\title{
The treatment analysis of the patients suffering from vaginismus and the correlation with the psychological issues
}

\author{
Didem Kurban ${ }^{1}$, Suleyman Eserdag ${ }^{1}$, Emrah Yakut ${ }^{2}$, Prabhu Chandra Mishra*
}

\begin{abstract}
${ }^{1}$ Hera Clinic Ankara for Cosmetic- Reconstructive Gynecology, Sexual Therapy and Wellness, Ankara, Turkey ${ }^{2}$ Department Memorial Ankara Hospital, Balgat Mah. Mevlana Blv. 1422. Sok. No: 4, 06520, Ankara, Turkey

${ }^{3}$ Head, StemMax Research and Therapeutics Pvt. Ltd., New Delhi, India
\end{abstract}

Received: 19 January 2021

Accepted: 02 March 2021

\section{*Correspondence: \\ Dr. Prabhu Chandra Mishra, \\ E-mail: info@stemgenn.com}

Copyright: ( ) the author(s), publisher and licensee Medip Academy. This is an open-access article distributed under the terms of the Creative Commons Attribution Non-Commercial License, which permits unrestricted non-commercial use, distribution, and reproduction in any medium, provided the original work is properly cited.

\section{ABSTRACT}

Background: We aimed to present the demographic information, treatment protocol, and results of 482 female patients that presented to our clinic specialized in sexual dysfunction with the complaint of no or only partial sexual intercourse and were diagnosed with primary vaginismus.

Methods: The female patients were asked eight questions about demographics; 13 questions about marriage; seven questions about family structure and upbringing; three questions about history of psychiatric diseases and general phobias; and 17 questions about sexual history and previous treatments. The male spouses were asked seven questions concerning age, occupation, educational level, personality, sexual experience, and sexual dysfunction.

Results: The median age of the female patients was 28 and their spouses was 29 . The mean duration of marriage was 18.2 months. Of the female patients, $65.4 \%$ reported that they felt they would have pain during sexual intercourse, $23.6 \%$ stated that they really had pain, $74.1 \%$ mentioned that they had heard horrifying stories about the first night of marriage in the pre-marital period. Cognitive behavioral therapy was performed alone in $85.7 \%$ of the patients, following hymenotomy in 5\%, and following hymenectomy in $9.3 \%$.

Conclusions: False and exaggerated information about sexuality being embedded in the subconscious of women is very effective in the development of vaginismus. On the other hand, traditional family structure, adolescent traumas, first night stories, and superstitions about sexuality are among the important causes of vaginismus.

Keywords: Female sexual pain, Genito-pelvic pain, Penetration disorder, Psychological issue in vaginismus treatment, Vaginismus, Vaginismus treatment

\section{INTRODUCTION}

Vaginismus is defined as involuntary contractions in the pelvic muscles in the outer third of the vagina during sexual intercourse, which makes penetration impossible or extremely painful. In vaginismus, the vaginal muscles that are normally under the complete control of women, strongly contract during sexual intercourse just before the penis enters the vagina, making penetration almost impossible. These symptoms are usually accompanied by avoidance of sexual intercourse. ${ }^{1,2}$ Furthermore, difficulties with vaginal penetration can also arise in cases other than sexual intercourse, such as gynecological examination, tampon placement, and insertion of foreign bodies into the vagina.

Vaginismus, which has been suggested to be rare in Western countries, is the most common problem among those who present to hospitals with the complaints of sexual dysfunction in Turkey. Vaginismus is the main problem in $43-75.9 \%$ of cases that apply to sexual therapy clinics. ${ }^{3-7}$ The prevalence of vaginismus 
worldwide is between 1 and $7 \%$, but reported to range from 5 to $17 \%$ in clinical conditions. ${ }^{89}$ In Turkey, it was found to be much higher in most studies, being reported as $41.7 \%$ by Oniz et al, $58.06 \%$ by Yasan et al, and $41 \%$ by Yildirim et al ${ }^{10-12}$ In contrast, in a study conducted in 2010 in Konya province, Yilmaz et al investigated sexual problems in the married population and calculated the rate of vaginismus as $15.3 \% .^{13}$

Vaginismus cases are classified as primary and secondary. In primary vaginismus (lifelong) cases, women have never experienced sexual intercourse in their lives while secondary (acquired) vaginismus refers to the loss of the ability to allow penetration due to sexual or genital trauma (eventful birth, miscarriage, abortion, rough gynecological examination, harassment or rape) despite the history of healthy sexual intercourse. The majority of cases of vaginismus encountered in the clinic are primary vaginismus ${ }^{14}$

Vaginismus is also an anxiety disorder. For this reason, it is also called 'sexual phobia' by some sexologists. ${ }^{15}$ The most common fears are related to the thoughts that the woman will have pain during sexual intercourse, the vagina is narrow, there is a wall inside the vagina, the vagina is closed, the first night will be difficult, painful, and bloody, the penis will penetrate the wrong organ, such as the bladder and anus, or the penis may be trapped inside the vagina. ${ }^{3}$ Some of the patients have such high anxiety that they fear dying during sexual intercourse. In vaginismus, there is no initial problem of sexual reluctance, although this may develop over time mostly due to the fear of pain and soreness. Sexual desire usually improves spontaneously when vaginismus is treated.,16

The first step in the diagnosis of vaginismus is a complete pelvic examination. This examination does not involve the use of a device and aims to provide information about the anatomical structure of the hymen and the severity of vaginismus. Although vaginismus is a psychological disorder, some anatomical reasons that complicate sexual intercourse can contribute to the development of this condition and make treatment difficult. A pelvic examination is necessary for the diagnosis of these possible anatomical causes. Hymen anomalies are one of the leading anatomical causes. In the presence of a septate hymen with a half-moon shape and high edge or rigid hymen, the removal of the physical barrier by hymenotomy or hymenectomy facilitates the treatment of vaginismus.

The main treatment of vaginismus is cognitive behavioral therapy (CBT) for sexual disorders. ${ }^{7,14}$ In this treatment, firstly, the patients' misconceptions about sexuality, the hymen, what happens on the first night, and female anatomy are corrected by providing them with accurate and factual information.

Then, the operation of the pelvic muscles is taken under control to eliminate involuntary contractions. A fundamental step in the treatment of vaginismus is to regenerate muscle memory and teach the patient how to control the pelvic muscles.

In this study, we aimed to present the demographic information, treatment protocol, and treatment outcome of 482 female patients that presented to our clinic with the complaint of no or only partial sexual intercourse and were diagnosed with primary vaginismus.

\section{METHODS}

A total of 482 female patients that presented to our private health center on female sexual function disorders in Ankara between 2016 and 2018 and were diagnosed with primary vaginismus based on sexual and medical history and pelvic examination findings were included in the study. A 48-item questionnaire prepared by the researchers was administered to the female patients and a seven-item questionnaire to their spouses.

First, the female patients were interviewed alone and asked eight questions related to demographics, such as age, occupation, educational level, marital status, parents' occupation, and number of siblings; 13 questions about marriage; seven questions about family structure and upbringing; three questions about previous psychiatric diseases and general phobias; and 17 questions about sexual history (orgasm, sexual desire, sexual myths, sexual traumas, sexual fears, etc.) and previous treatments. The male spouses were called into the room and were asked seven questions related to age, occupation, educational level, number of marriages, personality structure, sexual experience, and sexual dysfunction.

Then, the female patients were asked to lie on the gynecological examination table and their degree of vaginismus was determined using the Lamont classification. ${ }^{17}$ Hymen examination was undertaken to identify any anatomical or hymenal problem. The entry of the vagina was touched using a single finger, and if the patient permitted (Lamont grade 1), the finger was advanced into the vagina to evaluate the degree of contractions at the vaginal entrance and the flexibility of the hymen.

During the examination, the Q-tip test was performed in patients with the complaint of dyspareunia. The patients with vulvar vestibulitis syndrome and/or a vaginal infection were excluded from the study. In the presence of a septate hymen with a half-moon shape or a rigid hymen, hymenotomy was performed using local anesthesia, and in cases presenting with an almost imperforate hymen with a thick septum, hymenectomy was undertaken using sedation anesthesia. The patients that underwent hymenotomy continued CBT the following day, while those that underwent hymenectomy were called to the clinic for CBT after recovery (after four weeks on average). 
A total of 425 patients were included in the CBT program with their spouses. Of the remaining 57 patients, 52 were not accompanied by their husbands and five were single and did not have a partner. In the therapy, first, the male and female genital system anatomy, hymen structure, and sexual fears were discussed, and accurate and factual information about sexuality was provided. The misconceptions of the patients and their spouses, if present, were corrected, and sexual myths were dispelled. In each therapy session, approximately 20 minutes of cognitive therapy and 40 minutes of behavioral exercises were performed. The duration of this treatment was increased up to two hours in vaginismus cases with an advanced level of contraction and anxiety. The therapy was organized as one session per day and continued for three to seven consecutive days.

Behavioral exercises were performed under the supervision of psychologists and sexual therapists in the clinical environment. They consisted of general relaxation, breathing exercises, Kegel exercises, leg opening, looking at the genital area with a mirror, touching the genital area, perineum massage with cream and oils, and gradually progressing pelvic muscle exercises with dilators. Daily exercises were given to the patient as homework. They were asked to do the exercises at home first on their own and then with their spouses, if present.

Written informed consent was obtained from all participants. The study was approved by Memorial Ankara Hospital Ethical Review Committee.

Statistical analyses were performed using SPSS software version 24.0. Descriptive statistics were expressed as median for non-normally distributed variables.

\section{RESULTS}

The mean age of the 482 female patients included in the study was calculated as $28.4 \pm 5.4$ (range 18-54) years, and the mean age of their spouses was $30.8 \pm 5.8$ (range 20-62) years. The mean duration of marriage was 18.2 months, ranging from four days to 15 years (Table 1 ).

Table 1: Demographic characteristics of the patients.

\begin{tabular}{|llll|}
\hline Characteristic & & Female patients, $\mathbf{N}(\%)$ & Spouses, $\mathbf{N}(\%)$ \\
\hline Age (years) & Mean age $( \pm \mathrm{SD})$ & $28.4( \pm 5.4)$ & $30.8( \pm 5.8)$ \\
\hline \multirow{5}{*}{ Educational level } & Primary school & $12(2.5)$ & $15(3.5)$ \\
\cline { 2 - 4 } & Middle school & $31(6.4)$ & $35(8.2)$ \\
\cline { 2 - 4 } & High school & $115(23.8)$ & $125(29.3)$ \\
\cline { 2 - 4 } & University & $235(48.7)$ & $194(45.5)$ \\
\cline { 2 - 4 } & Master's degree & $56(11.6)$ & $37(8.6)$ \\
\cline { 2 - 4 } & PhD & $28(5.8)$ & $20(4.6)$ \\
\hline \multirow{3}{*}{ Occupation } & Unemployed/housewife & $181(37.5)$ & $4(0.9)$ \\
\cline { 2 - 4 } & Worker & $32(6.6)$ & $90(20.6)$ \\
\cline { 2 - 4 } & Civil servant & $135(28.0)$ & $159(36.4)$ \\
\cline { 2 - 4 } & Self-employed & $31(6.4)$ & $4(0.9)$ \\
\cline { 2 - 4 } & Academician & $5(1.0)$ & $90(20.6)$ \\
\cline { 2 - 4 } Country of residence & Private sector employee & $98(20.3)$ & \\
& Turkey & $438(90.8)$ & \\
\cline { 2 - 4 } & Other countries & $44(9.1)$ & \\
\hline
\end{tabular}

Table 2: Marital characteristics of the patients.

\begin{tabular}{|lll|}
\hline \multirow{3}{*}{ Characteristic } & & $\mathbf{N}(\%)$ \\
& Married & $456(94.6)$ \\
\cline { 2 - 3 } & Single & $21(4.3)$ \\
\cline { 2 - 3 } Type of marriage & Divorced & $5(1.0)$ \\
\hline \multirow{2}{*}{ Duration of knowing the spouse (days) (months) } & Dating & $425(88.8)$ \\
\cline { 2 - 3 } Duration of marriage (days) (months) & Arranged & $51(10.6)$ \\
\cline { 2 - 3 } \multirow{2}{*}{ Kin marriage } & Eloping & $6(0.6)$ \\
\hline & Median time (range) & $1440.0(60.0-9720.0) ; 48.0(2.0-324.0)$ \\
\hline
\end{tabular}


Table 3: General characteristics of the patients and their family structure.

\begin{tabular}{|c|c|c|}
\hline Characteristics & & $\mathbf{N}(\%)$ \\
\hline \multirow{3}{*}{ Type of upbringing } & Strict & $65(13.5)$ \\
\hline & Moderate & $325(67.5)$ \\
\hline & Relaxed & $92(18.9)$ \\
\hline \multirow{3}{*}{ Family structure } & Religious & $131(27.2)$ \\
\hline & Conservative & $295(61.2)$ \\
\hline & Modern & $56(11.6)$ \\
\hline \multirow{6}{*}{ Authority } & Mother & $145(30.0)$ \\
\hline & Father & $158(32.7)$ \\
\hline & Both & $112(23.2)$ \\
\hline & Brother & $4(0.8)$ \\
\hline & Others & $10(2.1)$ \\
\hline & None & $17(3.5)$ \\
\hline \multirow{2}{*}{ Familial history of vaginismus } & Absent & $428(88.8)$ \\
\hline & Present & $54(11.2)$ \\
\hline \multirow{2}{*}{ Told horrifying stories about the first night } & No & $88(18.2)$ \\
\hline & Yes & $358(74.1)$ \\
\hline \multirow{2}{*}{ Marital problems } & Absent & $398(82.4)$ \\
\hline & Present & $40(8.3)$ \\
\hline \multirow{2}{*}{ Reason for presentation to the clinic } & To solve the sexual problem & $456(94.7)$ \\
\hline & To have children & $26(5.3)$ \\
\hline \multirow{2}{*}{ Parents' knowledge of the problem } & Absent & $288(59.9)$ \\
\hline & Present & $194(40.1)$ \\
\hline \multirow{5}{*}{ Diagnosed psychological disorders** } & Anxiety disorder & $11(2.3)$ \\
\hline & Migraine & $29(6.0)$ \\
\hline & Obsessive compulsive & $5(1.0)$ \\
\hline & Panic attack & $11(2.3)$ \\
\hline & Depression & \\
\hline \multirow{15}{*}{ Fears $* *$} & Loneliness & $71(14.7)$ \\
\hline & Height & $139(28.8)$ \\
\hline & Dark & $99(20.5)$ \\
\hline & Needles & $118(24.4)$ \\
\hline & Blood & $55(11.4)$ \\
\hline & Cats & $94(19.5)$ \\
\hline & Dogs & $128(26.5)$ \\
\hline & Insects & $153(31.7)$ \\
\hline & Snakes & $176(36.4)$ \\
\hline & Enclosed spaces & $100(20.7)$ \\
\hline & Dentist & $51(10.6)$ \\
\hline & Sea & $22(4.6)$ \\
\hline & Gynecological examination & $227(47.0)$ \\
\hline & Swallowing pills & $42(8.7)$ \\
\hline & Other & $5(1.0)$ \\
\hline \multirow{9}{*}{ Source of first sexual information** } & Parents & $20(4.7)$ \\
\hline & Relatives & $67(15.7)$ \\
\hline & Books & $39(9.1)$ \\
\hline & Friends & $61(14.3)$ \\
\hline & Internet & $96(22.5)$ \\
\hline & Spouse & $12(2.8)$ \\
\hline & School & $47(11.0)$ \\
\hline & None & $34(7.9)$ \\
\hline & disorder & $18(3.7)$ \\
\hline
\end{tabular}

**Patients were allowed to select more than one option in this category.

The mean time from the time that the female patients met their spouses to the time of the study was 48 months, ranging from two months to 27 years (Table 2).
According to the responses to the questions about the family structure of the patients, $13.5 \%$ of the women described their upbringing as strict, $67.5 \%$ as moderate, and $18.9 \%$ as relaxed. Concerning the family history of vaginismus, $11.2 \%$ of the participants stated that there 
was a history of vaginismus in their family, while $74.1 \%$ confirmed that they had been told horrifying stories about the first night in their immediate surroundings before marriage. The female participants received the introductory information about sex from the internet at $22.5 \%$, from their relatives at $15.7 \%$, from their friends at $14.3 \%$, from their school at $11 \%$, from books at $9.1 \%$, from their parents at $4.2 \%$, and from their spouses at $2.8 \%$, while $7.9 \%$ stated that they had not received such information (Table 3).

As shown in Table 3, in addition to vaginismus, defined as vaginal penetration phobia, most of the participants also had other phobias related to gynecological examination, snakes, insects, and heights. While $94.7 \%$ of the patients stated that the primary reason for their presentation to the clinic was to solve their sexual problem, $5.3 \%$ wanted to have children, and only $40.1 \%$ stated that their parents had knowledge of their problems.

When the sexual misconceptions and myths of the patients were questioned, the female participants were found to believe the following: 'my vagina is too narrow' $(61.5 \%)$, 'the first night is extremely painful' (54.5\%), 'there is excessive bleeding on the first night' (52.8\%), 'the vagina is torn or ruptured on the first night' (37.5\%), 'the penis can accidentally penetrate the anus during sex' $(35.6 \%)$, 'the penis can accidentally penetrate the bladder' $(33.5 \%)$, and 'the penis can be trapped in the vagina', also known as penis captivus (34\%) (Table 4).

Of the female patients, $88.2 \%$ attended CBT with their spouses and $11.8 \%$ alone. When the 425 men who were the spouses of female patients that attended couples CBT were questioned about their previous sexual experience, $45.8 \%$ stated that they had previous sexual experience, $38.5 \%$ stated that they had no previous sexual experience, $15.7 \%$ stated that they had very little (once to four times in total) previous sexual experience. Concerning sexual problems, $77.4 \%$ of the 425 men stated that they did not have any sexual problem (Table 5). Concerning the history of coitus, $65.4 \%$ stated that they felt like they would have pain during sexual intercourse and $23.6 \%$ reported that they really felt pain. In their first anamnesis, $69.8 \%$ of the female participants stated that complete coitus never occurred, $14.3 \%$ stated that there was partial sexual penetration, $11 \%$ stated that they had complete coitus once, and 5\% stated that it was painful more than once (Table 4).

Table 4: Sexual anamnesis, examination findings and treatment characteristics.

\begin{tabular}{|c|c|c|}
\hline Variable & & $\mathbf{N}(\%)$ \\
\hline \multirow{9}{*}{ Myths** } & Penis captivus & $143(34.2)$ \\
\hline & Penis can accidentally penetrate the anus & $149(35.6)$ \\
\hline & First night is painful & $228(54.5)$ \\
\hline & First night is bloody & $221(52.8)$ \\
\hline & Vagina will be torn/ruptured on the first night & $157(37.5)$ \\
\hline & Coitus can result in death & $9(2.1)$ \\
\hline & Narrow vagina & $257(61.5)$ \\
\hline & Husband's penis is too large & $51(12.2)$ \\
\hline & Penis can penetrate the bladder & $140(33.5)$ \\
\hline \multirow{9}{*}{ Sexual trauma** } & Abuse (once or more than once) (by a relative, someone close, or someone unknown) & $50(10.3)$ \\
\hline & Rape & $1(0.2)$ \\
\hline & Incest & $1(0.2)$ \\
\hline & Watching a video of childbirth and being scared & $58(12.0)$ \\
\hline & Physical violence & $13(2.7)$ \\
\hline & Genital trauma history & $14(2.9)$ \\
\hline & Watching a pornographic film and being disgusted by it & $70(14.5)$ \\
\hline & Witnessing the sexual intercourse of parents (hearing-seeing) & $68(14.1)$ \\
\hline & Wetting the bed as a child (over five years old) & $64(13.2)$ \\
\hline \multirow{15}{*}{$\begin{array}{l}\text { Perceived cause } \\
\text { of the problem** }\end{array}$} & First night stories & $263(61.3)$ \\
\hline & Type of upbringing & $150(34.9)$ \\
\hline & Like my comfort & $295(68.7)$ \\
\hline & Marital problems & $16(3.7)$ \\
\hline & Inexperienced spouse & $77(16.3)$ \\
\hline & Shy & $97(22.6)$ \\
\hline & Incompatible with the spouse & $7(1.6)$ \\
\hline & Length of flirting & $26(6.0)$ \\
\hline & Inadequately informed & $200(46.6)$ \\
\hline & Problems around the time of marriage & $22(5.1)$ \\
\hline & Abuse & $21(4.9)$ \\
\hline & Fear of becoming pregnant & $39(9.1)$ \\
\hline & Fear of growing up & $19(4.4)$ \\
\hline & Physical genital trauma & $1(0.2)$ \\
\hline & Looking ugly during intercourse & $10(2.0)$ \\
\hline
\end{tabular}




\begin{tabular}{|c|c|c|}
\hline \multicolumn{2}{|l|}{ Variable } & $\mathbf{N}(\%)$ \\
\hline \multirow{6}{*}{ Clitoral orgasm } & Difficult gynecological examination-intervention & $8(1.8)$ \\
\hline & Witnessing parents' intercourse (hearing-seeing) & $16(3.7)$ \\
\hline & No idea & $50(11.6)$ \\
\hline & Absent & $131(27.1)$ \\
\hline & Present & $323(67.1)$ \\
\hline & Reduced & $28(5.8)$ \\
\hline \multirow{3}{*}{ Sexual desire } & Absent & $25(5.2)$ \\
\hline & Normal & $342(71.0)$ \\
\hline & Reduced & $115(23.8)$ \\
\hline \multirow{2}{*}{ Feeling of pain } & Real pain & $114(23.6)$ \\
\hline & Feeling it will be painful & $316(65.4)$ \\
\hline \multirow{4}{*}{ Coitus } & Never had a complete coitus & $336(69.8)$ \\
\hline & Only partial coitus & $69(14.2)$ \\
\hline & Complete coitus once & $53(11.0)$ \\
\hline & Once or more than once painful coitus & $24(5.0)$ \\
\hline \multirow{11}{*}{$\begin{array}{l}\text { Previous } \\
\text { attempts to } \\
\text { resolve } \\
\text { vaginismus** }\end{array}$} & Alcohol & $154(40.7)$ \\
\hline & Religious hodjas & $79(20.9)$ \\
\hline & Lubricator & $309(81.7)$ \\
\hline & Anesthetic cream & $167(44.2)$ \\
\hline & Tranquilizer & $121(32.0)$ \\
\hline & Muscle relaxant & $128(33.8)$ \\
\hline & Watching pornography & $127(33.6)$ \\
\hline & Anesthesia from the waist down & $4(1.0)$ \\
\hline & Hypnosis & $7(1.8)$ \\
\hline & EMDR & $1(0.2)$ \\
\hline & Dilator & $28(7.4)$ \\
\hline \multirow{5}{*}{ Hymen } & Intact annular & $271(56.4)$ \\
\hline & Intact septate & $11(2.2)$ \\
\hline & Intact slightly raised & $11(2.2)$ \\
\hline & Intact raised & $47(9.8)$ \\
\hline & Imperforate & $142(29.4)$ \\
\hline \multirow{4}{*}{$\begin{array}{l}\text { Examination } \\
\text { findings }\end{array}$} & Lamont 1 & 88 (21.6) \\
\hline & Lamont 2 & $158(38.8)$ \\
\hline & Lamont 3 & $148(36.4)$ \\
\hline & Lamont 4 & $13(3.2)$ \\
\hline \multirow{4}{*}{$\begin{array}{l}\text { Previous non- } \\
\text { surgical } \\
\text { procedures** }\end{array}$} & Absent & $146(43.6)$ \\
\hline & Present (finger exercise) & $67(20.0)$ \\
\hline & Therapy & $96(28.6)$ \\
\hline & Gynecological examination & $174(51.9)$ \\
\hline \multirow{4}{*}{$\begin{array}{l}\text { Previous } \\
\text { surgical } \\
\text { procedures }\end{array}$} & Absent & $467(96.6)$ \\
\hline & Present & $16(3.3)$ \\
\hline & Hymenectomy & $9(1.9)$ \\
\hline & Hymenotomy & $7(1.4)$ \\
\hline \multirow{3}{*}{$\begin{array}{l}\text { Treatment } \\
\text { applied }\end{array}$} & Cognitive behavioral therapy (CBT) & $413(85.7)$ \\
\hline & CBT + hymenetomy under local anesthesia & $24(5.0)$ \\
\hline & CBT + hymenectomy under general anesthesia & $45(9.3)$ \\
\hline
\end{tabular}

**Patients were allowed to select more than one option in this category.

Table 5: Characteristics of the patients' spouses.

\begin{tabular}{|c|c|c|}
\hline \multicolumn{2}{|l|}{ Characteristic } & $\mathbf{N}(\%)$ \\
\hline \multirow{4}{*}{ Type of personality } & Oppressive-conservative & $12(2.5)$ \\
\hline & Nervous-angry & $51(10.6)$ \\
\hline & Extremely understanding-patient & $361(74.7)$ \\
\hline & Prone to violence & $9(1.9)$ \\
\hline \multirow{3}{*}{ Sexual experience } & Absent & $130(38.5)$ \\
\hline & Present & $155(45.8)$ \\
\hline & Little & $53(15.7)$ \\
\hline Sexual problems*** & Absent & $314(77.4)$ \\
\hline
\end{tabular}

Continued. 


\begin{tabular}{|llc|}
\hline Characteristic & N (\%) \\
\cline { 2 - 3 } & Present & $111(22.6)$ \\
\cline { 2 - 3 } & Primary erectile dysfunction & $4(0.94)$ \\
\cline { 2 - 3 } & Secondary erectile dysfunction & $22(5.1)$ \\
\cline { 2 - 3 } & Primary premature ejaculation & $21(4.9)$ \\
\cline { 2 - 3 } & Secondary premature ejaculation & $41(9.6)$ \\
\cline { 2 - 3 } & Primary lack of sexual drive & $2(0.47)$ \\
\cline { 2 - 3 } & Secondary lack of sexual drive & $22(5.1)$ \\
\cline { 2 - 3 } & Late ejaculation & $1(0.2)$ \\
\hline
\end{tabular}

**Participants were allowed to select more than one option in this category.

When asked about previous treatments, $43.6 \%$ of the patients stated that they had never received any previous treatment, $20 \%$ said they tried the finger exercise, and $28.6 \%$ reported that they had previously attended therapy. It was determined that nine patients $(1.9 \%)$ had previously undergone hymenectomy in different clinics and seven $(1.4 \%)$ had undergone hymenotomy, but their vaginismus problem persisted. Of the female patients, 85.7\% underwent CBT alone, 5\% underwent CBT after hymenotomy under local anesthesia, and $9.3 \%$ underwent CBT after hymenectomy under general anesthesia (Table 5). The majority of the patients $(80.2 \%)$ completed CBT in three sessions, followed by $13.7 \%$ of patients in five sessions, and $6.1 \%$ in seven sessions.

\section{DISCUSSION}

The prevalence of vaginismus can vary greatly in studies conducted in different cultural societies. The proportion of vaginismus in the general population is between 3 and $13 \%$, and there are studies reporting it to range from 21 to $48 \%$ among sexual dysfunctions presenting to the clinic. ${ }^{18}$ In a study conducted in Iran, it was reported that $8 \%$ of 300 married women who presented to a family planning clinic in Tehran were diagnosed with vaginismus. ${ }^{19}$ In studies conducted in Western countries, vaginismus is one of the leading female sexual dysfunctions, although it mostly presents as a reduced sexual desire..$^{20}$ In their study with similar characteristics to our research, Özdemir et al evaluated 449 married couples with no complete coitus, who presented to the sexual health clinics in Istanbul, and found that the most prominent cause was vaginismus in $81 \%$, erectile dysfunction in $10.5 \%$, and premature ejaculation in $5 \%$. The patients presenting with the complaint of a lack of sexual intercourse accounted for $24 \%$ of all patients admitted to their clinic. ${ }^{21}$

It was seen that the educational level of the participants in our study was higher than the normal population, with $66.1 \%$ of the female patients and $58.7 \%$ of their spouses being university graduates or having a higher degree. Similarly high educational levels were reported in the studies of Akhavan-Taghavi et al and Tuğrul et al.,22 Since these results represent only the patients that applied to our clinic, not the general population, it is not possible to state that vaginismus is a problem of women with a high level of education. However, it can be inferred that it is easier for women with higher education to seek treatment and access specialized clinics. As a result of the widespread use of the internet and the increase in posts in social media, more individuals with a higher educational level now research their health problems on these platforms, which we consider increases the preference for attending private clinics. It is also likely that women with a low level of education are shy about consulting another person about their sexual problems. ${ }^{7,23}$

In the current study, $65.4 \%$ of the patients stated that they felt like they would experience pain during sexual intercourse, and $23.6 \%$ stated that they really felt pain. In a study conducted by Oktay et al with 200 women with vaginismus, only $18 \%$ of the patients reported excessive pain at first coitus while $57 \%$ stated that they experienced fear of pain. ${ }^{5}$

Phobic reactions play an important role in the diagnosis of vaginismus. Due to the presence of phobias related to gynecological examination, snakes, insects, heights, and dogs in the majority of our patients, it can be considered that vaginismus was also a phobic reaction in these individuals and their pain thresholds might be lower. The ability of most patients to have sexual intercourse after treatments, such as gradual exposure and relaxation exercises to reduce fear and anxiety is evidence that fear and anxiety play an important role in the etiology of vaginismus. ${ }^{24}$

In a study of 77 women diagnosed with vaginismus, it was found that $47 \%$ of these women had fear of vaginal penetration, $10 \%$ were disgusted by the idea, and $13 \%$ experienced both fear and disgust. ${ }^{25}$ Oktay et al evaluated 200 women diagnosed with vaginismus and reported the presence of fear of soreness and excessive pain in $57 \%$ of these patients, fear of excessive bleeding in $31 \%$, fear of tearing/rupture in $18 \%$, fear of being left/locked in the vagina in $17 \%$, fear of fainting/dying in $11 \%$, and feeling of filthiness/disgust in $9 \% .5$ In our study, the misconceptions of women with vaginismus related to sexuality were a narrow vagina in $61.5 \%$, pain on the first night in $54.5 \%$, extreme bleeding on the first night in $52.8 \%$, and torn/ruptured vagina on the first night in $37.5 \%$, which is consistent with the literature. Another fear of these patients related to sexual intercourse was that the penis would penetrate the wrong organ and harm them. The fact that most of the male spouses were inexperienced or had little experience in coitus may have also increased the women's anxiety. In our study, 35.6\% of the patients thought that the penis could penetrate the anus and $33.5 \%$ thought that it could enter the bladder. 
The causes of vaginismus can be evaluated under psychological and physiological headings. We consider that in most of the patients, vaginismus has a psychological cause; e.g., misconceptions or limited knowledge about the hymen, vagina, and sex, protective parents, perfectionist personality, high self-control, moral and religious pressures, history of sexual harassment or unpleasant memories related to sex, and fear of the first night. The first sexual experience is also very important. In $12.3 \%$ of our patients, the first coitus was so painful that the patients did not engage in further sexual intercourse. We associate this with the negative reinforcement. During the first coitus, pain can be experienced due to the hard and thick structure of the hymen or excessive contraction.

Only small amount of vaginismus cases have physiological factors that trigger vaginismus. Hymen anomalies are the most common anatomical causes of vaginismus. A raised, septate or thick hymen makes sexual intercourse difficult or impossible. In this study, hymen anomalies which made coitus difficult were detected in $14.3 \%$ of women and a surgical intervention was performed (Table 4). However, surgical methods should be performed only if there is an anatomical obstacle related to hymen. Otherwise, only in the presence of psychological reasons, the patient's anxiety is further increased, and the problem may continue even after the removal of the hymen. In addition, it is important to offer CBT to patients that undergo surgery for hymen anomalies in order to overcome the negative conditioning acquired over time.

The reasons for the development of vaginismus also differ in different societies. In Western societies, less emphasis on premarital virginity, provision of sexual education in schools, and the increasingly abandoned over-interfering and dependent mother model in child rearing are considered as the lower prevalence of vaginismus compared to Eastern cultures. ${ }^{26}$ The lack of sexual education, women not having knowledge of their own genitalia, excessive meaning attributed to the concept of virginity, direct experience with coitus without having prior gradual experience with sexual intercourse, and taboos in the understanding of general sexuality are among the causes of more common vaginismus in Eastern cultures. $^{27}$

Only $11 \%$ of the women that participated in our study stated that they had obtained their first sexual information from their school and $4.2 \%$ received information from their parents. This situation clearly shows how inadequate sexual education is in Turkey. The most important factor in reducing the risk for vaginismus is the provision of sexual education by the family and school from a very early age because people that are inadequately informed or misinformed have the highest risk of vaginismus and other sexual problems in the years to come. ${ }^{28}$

Ward and Ogden evaluated the personal explanations of women with vaginismus about the possible causes of this condition and reported that for 67 women with the current complaint of vaginismus and 22 with the history of such a complaint, 'growing up with the idea that sex is wrong' was the second most common reason for the development of vaginismus. ${ }^{29}$ Similarly, the diagnosis of vaginismus in Tehran was mostly associated with the lack of information/misinformation about sexuality. ${ }^{19}$ In our study, the responses of the female participants to the question, 'Why do you think you have this problem?' were similarly related to the stories they had heard about the first night $(61.3 \%)$, inadequate knowledge $(46.6 \%)$, upbringing $(34.9 \%)$, and the lack of experience of their spouses $(16.3 \%)$. Furthermore, $74.1 \%$ of our patients stated that they had been told horrifying stories about the first night in their immediate surroundings prior to marriage.

In this study, the mean time from marriage to presentation was 18.2 months, which is consistent with the literature. In previous studies, the time from marriage to presentation to the clinic was reported as 26 months in a study by Munasinghe et al conducted in 2004 in Sri Lanka and 15 months by Doğan et al in their 2008 study in Turkey. ${ }^{7,30}$ In the current study, it is seen that there were marriages without coitus for up to 15 years. The reason for this may be the patients feeling embarrassed to share their problem with someone else, waiting for the spontaneous recovery of their issue, previous unsuccessful treatment attempts, not knowing that vaginismus is a curable condition, and not being aware of the presence of specialized clinics on sexual disorders, or a combination of a few of these factors. On the other hand, the excessive tolerance of the male spouses, their ignorance of the problem, and thinking that their spouses could never overcome the problem with treatment may have delayed seeking professional support.

Although $82.4 \%$ of the participants stated that there was no other problem in their marriages, the problems experienced in their sexual lives are likely to turn into marital problems in the future and result in divorce. ${ }^{31}$ According to one study, more than $60 \%$ of couples that applied to the court for divorce stated that they had unsatisfactory sexual intercourse with their spouses. ${ }^{32}$

\section{CONCLUSION}

Early treatment of vaginismus before it progresses into a chronic problem is also considered to reduce the likelihood of secondary sexual problems, such as lack of sexual drive-in couples, premature ejaculation and erectile dysfunction in men, and the development of psychiatric problems, such as anxiety and depression. First, from an early age, age-appropriate sexual information should be provided to prevent the development of such problems. Second, early diagnosis and treatment should be undertaken to prevent the development of different sexual and psychiatric problems over time and improve the marriage relationship. Considering the very high success rates in patients that comply with treatment, it is crucial to increase awareness of vaginismus and its treatable nature, and refer patients to specialized clinics. 
Funding: No funding sources

Conflict of interest: None declared

Ethical approval: The study was approved by the Memorial Ankara Hospital Ethical Review Committee

\section{REFERENCES}

1. American Psychiatric Association. Diagnostic and statistical manual of mental disorders. 4th edn. Text revision. Washington, DC: American Psychiatric Association; 2000.

2. Butcher J. ABC of sexual health: female sexual problems II: sexual pain and sexual fears. BMJ. 1999;318(7176):110-2.

3. Dogan S. Vaginismus and accompanying sexual dysfunctions in a Turkish clinical sample. J Sex Med. 2009;6(1):184-92.

4. İncesu C, Yetkin N. Assessment of 200 Subjects referred to a sexual dysfunction outpatient clinic in Turkey. In: Proceedings of the XIIIth. World Congress of Sexology; 1997:285-290.

5. Oktay M, Tombul K. Two hundred cases of vaginismus: their and their husbands' psychological evaluation. New Sympos. 2003;41:115-9.

6. Sungur M. Evaluation of couples referred to a sexual dysfunction unit and prognostic factors in sexual and marital therapy. J Sex Marital Ther. 1994;9:251-65.

7. Tugrul C, Kabakçi E. Vaginismus and its correlates. J Sex Marital Ther. 1997;12:23-34.

8. Konkan R, Bayrak M, Gönüllü OG, Şenormancı O, Sungur MZ. Sexual function and satisfaction of women with vaginismus. Düşünen Adam: J Psychiatr Neurol Sci. 2012;25(4):305-11.

9. Lahaie MA, Boyer SC, Amsel R, Khalife S, Binik YM. Vaginismus: a review of the literature on the classification/diagnosis, etiology and treatment. Wom Health. 2010;6(5):705-19.

10. Oniz A, Keskinoglu P, Bezircioglu I. The prevalence and causes of sexual problems among premenopausal Turkish women. J Sex Med. 2007;4:1575-81.

11. Yasan A, Gurgen F. Marital Satisfaction, sexual problems and the possible diffuculties on sex therapy in traditional Islamic culture. J Sex Marital Ther. 2009;35:68-75.

12. Yıldırım EA, Akyüz F, Hacıoğlu M, Eşsizoğlu A, Çakmak AC, Çakmak E, et al. Relationship between presentation complaint and clinical diagnosis in patients admitted to sexual dysfunction clinic. Arch Neuropsychiatr. 2011;48(1):24-30.

13. Yilmaz E, Zeytinci IE, Sarı S, Karababa IF, Cilli AS, Kucur R. Investigation of sexual problems in married people living in the center of Konya. Turk Psikiyatri Derg. 2010;21(2):126-34.

14. Munasinghe T, Goonaratna C, de Silva P. Couple characteristics and outcome of therapy in vaginismus. Ceylon Med J. 2004;49:54-7.

15. Kaplan HS. The new sex therapy: active treatment of sexual dysfunctions. New York: Brunner-Mazel; 1974:413-429.
16. Beck JG. Vaginismus. In: O'Donohue W, Greer JH, eds. Handbook of sexual dysfunctions: assessment and treatment. Boston: AUyn and Bacon Inc.; 1993:381-397.

17. Lamont JA. Vaginismus: a reflex response out of control. Contemp Obstet Gynecol. 1994;3:30-2.

18. Ohl LE. Essentials of female sexual dysfunction from a sex therapy perspective. Urol Nurs. 2007;27(1):57-63.

19. Shokrollahi P, Mirmohamadi M, Mehrabi F, Babaei G. Prevalence of sexual dysfunction in women seeking services at family planning centers in Tehran. J Sex Marital Ther.1999;25:211-5.

20. Bachmann G. Female sexuality and sexual dysfunction: Are we stuck on the learning curve. J Sex Med. 2006;3:639-45.

21. Ozdemir O, Simsek F, Ozkardes S, Incesu C, Karakoç B. The unconsummated marriage: its frequency and clinical characteristics in a sexual dysfunction clinic. J Sex Marital Ther. 2008;34(3):268-79.

22. Akhavan-Taghavi MH, Asghari-Moghaddam MA, Froutan SK, Jadid-Milani M. Vaginismus in Iran: a single center report of 7 years' experience. Nephrourol Mon. 2015;7(6):e30077.

23. Kaplan HS. The new sex therapy: active treatment of sexual dysfunctions. Illustrated ed. Levittown, PA: Brunner/Mazel; 1974.

24. ter Kuile MM, van Lankveld JJ, de Groot E, Melles $\mathrm{R}$, Neffs J, Zandbergen M. Cognitive-behavioral therapy for women with lifelong vaginismus: Process and prognostic factors. Behav Res Ther. 2007;45(2):359-73.

25. Kaneko K. Penetration disorder: dyspareunia exists on the extension of vaginismus. J Sex Marital Ther. 2001;27:153-5.

26. Hiller J. Defining vaginismus. Sex Relat Ther. 2000;15:87-90.

27. Vaginismus YN. Sexual Dysfunctions Monograph Series. 1999;3:26-9.

28. Özmen E. Sexual myths and sexual dysfunctions. World Psychiatr. 1999;2:49-53.

29. Ward E, Ogden E. Experiencing vaginismus sufferers' beliefs about causes and effects. Sex Marital Ther.1994;9:33-45.

30. Dogan S, Dogan M. The frequency of sexual dysfunctions in male partners of women with vaginismus in a Turkish sample. Int $\mathbf{J}$ Impot Res. 2008;20(2):218-21.

31. Aghamohmadiyan $\mathrm{H}$, Rezagholizade $\mathrm{T}$, Avazi M, Poshtiban H, eds. Satisfaction of sexual fanction in family and its coloration with divorce. First Congress in Family and Sexual Dysfunction. Shahed University, Tehran, Iran; 2003.

32. Mahani SA, Motlagh NS, Mahdavi VM, Hadian M, Lari AM. Involvement of families from Tehran to astonishing levels of health costs and relating factors: Justice Evaluation project in Tehran (Urban HEART 2010). Razi Med Sci J. 2014;21(126):15-26.

Cite this article as: Kurban D, Eserdag S, Yakut E, Mishra PS. The treatment analysis of the patients suffering from vaginismus and the correlation with the psychological issues. Int J Reprod Contracept Obstet Gynecol 2021;10:1328-36. 